\title{
Conversations with a pediatric psychiatrist
}

\author{
Interview with Dr Javeed Sukhera
}

\author{
Han Yan (Meds 2017), Ramona Neferu (Meds 2018) \\ Faculty Reviewer: Dr Javeed Sukhera, MD, FRCPC (Department of Psychiatry)
}

In the first of three interviews in this issue, we speak to Dr Javeed Sukhera. Dr Sukhera is an Assistant Professor in the Department of Psychiatry and Senior Designate Physician Lead for Child and Adolescent Psychiatry at London Health Sciences Centre/Victoria Hospital. He is also the Academic Director of the Global Health Curriculum at Schulich. Dr Sukhera has previously served for two years on the board of directors of the Association of American Medical Colleges.

UWOMJ: Tell us about yourself, specifically your education and career.

Javeed Sukhera: I am originally from Toronto and did my undergraduate training there. I took the opportunity to study medicine in Israel, which is where I was able to further explore my interests in global health. I studied at the Medical School for International Health, an innovative collaboration between Columbia University and Ben-Gurion University in Israel. During my fourth year, I returned to Canada for an elective in adolescent psychiatry and that is what sparked my interest in psychiatry. Prior to the elective, I was was close to pursuing family medicine, but inevitably chose psychiatry. I then completed my residency in Rochester, New York, as well as my fellowship. Two years ago, I decided to return to Canada, and I came to London, Ontario.

What made you interested in pediatric psychiatry? What makes this field unique?

I think that psychiatry is very unique because it is so different from every other field in medicine. All throughout medical school, you are taught to think a certain way. In psychiatry, you have to unlearn the need to always try to find a solution or a cure. There is not always a medication or a quick fix within psychiatry, and often, there is the opportunity for different methods of patient management. Pediatric psychiatry is especially interesting because you are working with a population during a developmentally sensitive period that can have a long-lasting impact on the patient's life.

Tell us about your other academic or personal interests and how you balance that.

Between clinic, research and academic teaching responsibilities, I would roughly estimate that 40 percent of my time is clinical, 40 academic and 20 research. I was recently awarded a grant to conduct research on unconscious biases within medicine and how they affect patient outcomes. Due to my appointment, I also have several academic responsibilities. For example, I am the Academic Director of Global Health Curriculum at Schulich. At the Office of Global Health, we have worked hard to integrate global health and public health topics into all four years of the undergraduate curriculum. Specific examples include the cultural competence workshop in your Population Health course and a Health Equity Panel in the Healthcare Systems course.

Global health is something that is very important to me and is an important part of my career. The old version of global health involved going abroad and providing healthcare in countries outside of Canada. Over time, we have recognized the problem of medical tourism, but also recognize that there is a benefit to learning medicine in different cultural environments. More recently, global health is more about the patients sitting in front of us, especially in Canada, and how understanding local and global contexts helps us understand the social and cultural determinants affecting this specific patient's care. Many students struggle with the idea of global health in a local setting, but the same principles relating to cultural competency apply both locally and globally.

How would you compare psychiatry to neurology or neurosurgery? What are the similarities or differences that you see? Do you often work in interdisciplinary teams?

I would say that neurology is somewhere between psychiatry and neurosurgery. We definitely work with neurology on certain cases. The problems that we see are often biosocial in nature, and there are referrals that flow in both directions. I would think of neurology as a field that deals with the brain whereas psychiatry is a field that deals with the mind. It is on rare occasions that we might work with the neurosurgeons. More often than not, we might be consulted by neurosurgery. The main difference for psychiatry that makes it unique from other specialties is that psychiatric complaints are multifaceted and very complex. Just this morning, I saw a boy with attention deficit hyperactivity disorder (ADHD) - a very bright boy. Early on, he had acknowledged how he might have been different and he has done well working with me. Our conversation was not limited to his medication, but also the social aspects of his illness. When someone has ADHD, it affects many parts of his life, such as his education and friends, just as some examples. My treatment extended beyond offering medication and included providing the opportunity to openly speak about the challenges of being a teenager who may need to rely on medication while trying to fit in with his peers.

Tell us about your family, or hobbies you have outside of work.

I am very lucky to have two beautiful children with my wife. We all enjoy travelling, but my favourite hobby would probably be just doing nothing on a Sunday afternoon. 


\section{Do you have a memorable case to share?}

There are just so many cases that are memorable. This one case that I remember is actually from when I was a fourth-year medical student. The patient was an adolescent male who adhered to Orthodox Jewish practices. He had very specific requests for his psychiatric care. For example, he refused to be touched by the female nurse. At the time, my supervisor was actually also a rabbi, and he was able to communicate genuinely with the patient. His approach showed a lot of patience and understanding. In the end, the solution that was finally agreed upon was for the patient to receive care from the nurse, who would be able to touch him with gloves on.

What do you see as the challenges/innovations within psychiatry in the next 20 years?

I think a current challenge is the bias within medicine that a lot of my patients face. You would be surprised at some of the comments that I hear from other physicians that stigmatize patients with psychiatric illness. The treatment that a lot of psychiatric patients receive is simply unfair and stems from ignorance. Especially in emergency rooms, these patients often face a lot of discrimination.

With psychiatry, there is also a great need to advocate for my patients. There are essential and vital medications or treatments that are not available everywhere. For example, some of my patients from more rural locations do not have access to a psychiatrist near them. Can you imagine that for other illnesses? For a diabetic not to have access to insulin or a heart failure patient to not be able to be pay for ACE inhibitors? It would be unheard of! Within psychiatry, there is a lot more for us to advocate for. This is a real challenge that I unfortunately see in my practice. Moving forward, there is a lot of room for improvement.

I see a lot of changes upcoming in psychiatry, especially with new psychotherapies, and psychopharmacological treatments that are being researched. So, there are some positive things to look forward to as well.

\section{What are your thoughts about the new Diagnostic and Statistical Manual of Mental Disorders (DSM) V?}

I am not a huge fan of the DSMs because I find that their categorizations oversimplify the problems that we are presented with. The DSM is helpful for providing guidelines for diagnosis, but most of our patients do not fit neatly into the categories that the DSM provides. I prefer to address the person in front of me instead of treating a formulaic diagnosis provided by the DSM.

\section{If a medical student is interested in psychiatry, do you have any advice for them?}

I would just say that there are many different facets to psychiatry. Pediatric psychiatry, for example, is very different. There is a lot to experience and see in psychiatry. Use the clerkship psychiatry rotation to explore and be open minded. You may be surprised at what interests you and what field you can imagine yourself practicing in. 